\title{
Simple Method for Quantification of Gadolinium Magnetic Resonance Imaging Contrast Agents Using ESR Spectroscopy
}

\author{
Keizo Takeshita,* Shota Kinoshita, and Shoko Okazaki \\ Laboratory of Analytical Chemistry, Faculty of Pharmaceutical Sciences, Sojo University; Kumamoto 860-0082, \\ Japan. Received June 22, 2011; accepted October 5, 2011; published online October 13, 2011
}

To develop an estimation method of gadolinium magnetic resonance imaging (MRI) contrast agents, the effect of concentration of Gd compounds on the ESR spectrum of nitroxyl radical was examined. A solution of either 4-oxo-2,2,6,6-tetramethylpiperidine- $N$-oxyl (TEMPONE) or 4-hydroxy-2,2,6,6-tetramethylpiperidine- $N$-oxyl (TEMPOL) was mixed with a solution of Gd compound and the ESR spectrum was recorded. Increased concentration of gadolinium-diethylenetriamine pentaacetic acid chelate (Gd-DTPA), an MRI contrast agent, increased the peak-to-peak line widths of ESR spectra of the nitroxyl radicals, in accordance with a decrease of their signal heights. A linear relationship was observed between concentration of Gd-DTPA and line width of ESR signal, up to approximately $50 \mathrm{mmol} / \mathrm{L}$ Gd-DTPA, with a high correlation coefficient. Response of TEMPONE was 1.4-times higher than that of TEMPOL as evaluated from the slopes of the lines. The response was slightly different among Gd compounds; the slopes of calibration curves for acua[ $N, N$-bis[2-[(carboxymethyl)[(methylcarbamoyl)methyl]amino]ethyl]glycinato(3-)]gadolinium hydrate (Gd-DTPA-BMA) $(6.22 \mu \mathrm{T} \cdot \mathrm{L} / \mathrm{mmol})$ and gadolinium-1,4,7,10-tetraazacyclododecane-1,4,7,10-tetraacetic acid chelate (Gd-DOTA) $(6.62 \mu \mathrm{T} \cdot \mathrm{L} / \mathrm{mmol})$ were steeper than the slope for Gd-DTPA $(5.45 \mu \mathrm{T} \cdot \mathrm{L} / \mathrm{mmol})$, whereas the slope for gadolinium chloride $(4.94 \mu \mathrm{T} \cdot \mathrm{L} / \mathrm{mmol})$ was less steep than that for Gd-DTPA. This method is simple to apply. The results indicate that this method is useful for rough estimation of the concentration of Gd contrast agents if calibration is carried out with each standard compound. It was also found that the plot of the reciprocal square root of signal height against concentrations of contrast agents could be useful for the estimation if a constant volume of sample solution is taken and measured at the same position in the ESR cavity every time. mation

Key words magnetic resonance imaging contrast agent; gadolinium; ESR; nitroxyl radical; quantitative esti-

Magnetic resonance imaging (MRI) is a powerful tool for diagnosis of various diseases including cancer and vasculopathy. Paramagnetic compounds such as Gd chelate compounds are usually used as contrast agents to identify the region of a lesion. New contrast agents are continually developed to improve the specificity of diagnosis and to lessen side effects, although gadolinium-diethylenetriamine pentaacetic acid chelate (Gd-DTPA), gadolinium-1,4,7,10-tetraazacyclododecane-1,4,7,10-tetraacetic acid chelate (Gd-DOTA), and their derivatives, such as acua[ $N, N$-bis[2-[(carboxymethyl)[(methylcarbamoyl)methyl]amino]ethyl]glycinato(3-)]gadolinium hydrate (Gd-DTPA-BMA), have been extensively used ${ }^{1-3)}$ (Fig. 1). Amounts and concentrations of Gd contrast agents have been determined by atomic absorption spectrometry, ${ }^{4}$ inductively coupled plasma atomic emission spectroscopy (ICPAES), ${ }^{5,6)}$ and inductively coupled plasma mass spectrometry (ICP-MS). ${ }^{6}$ They are probably, at present, the only methods to estimate Gd contrast agents, although quantification of the contrast agents is necessary to evaluate the agents synthesized and to monitor concentration of the agents in the blood circulation. Therefore, ESR method for the estimation of Gd contrast agents was investigated by utilizing the paramagnetism of Gd(III).

Although Gd(III) is a paramagnetic species, direct detection of Gd chelate compounds is difficult with ESR because the ESR relaxation time of Gd(III) is usually very short. Therefore, an estimation method was developed by utilizing interaction of $\mathrm{Gd}(\mathrm{III})$ with nitroxyl radicals. Nitroxyl radicals are durable and detectable with ESR. Paramagnetic species near nitroxyl radicals shorten spin-spin relaxation time $\left(T_{2}\right)$ of the nitroxyl radicals, resulting in the broadening of ESR signal of the radicals. This interaction of nitroxyl radical has been observed in concentrated solutions of potassium ferricyanide, chromium oxalate, and chelate complexes of $\mathrm{Ni}$ (II) and $\mathrm{Cu}(\mathrm{II}) .^{7-9)}$ In this study, a simple method for estimation of Gd contrast agents was developed, on the basis of spin-spin relaxation of nitroxyl radicals, and its ability was evaluated with two nitroxyl radicals, 4-oxo-2,2,6,6-tetramethylpiperidine $N$-oxyl (TEMPONE) and 4-hydroxy-2,2,6,6-tetramethylpiperidine $N$-oxyl (TEMPOL) (Fig. 1$)$.

\section{Experimental}

Reagents TEMPONE was purchased from Tokyo Chemi-

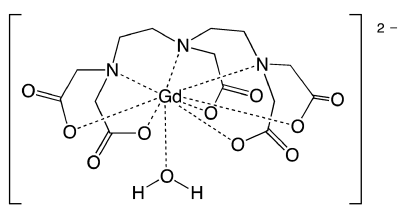

Gd-DTPA ( [Gd(DTPA) $\left.\left.\left(\mathrm{H}_{2} \mathrm{O}\right)\right]^{2-}\right)$

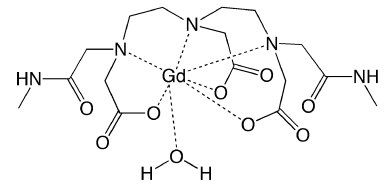

Gd-DTPA-BMA ( [Gd(DTPA-BMA) $\left.\left(\mathrm{H}_{2} \mathrm{O}\right)\right]$ )

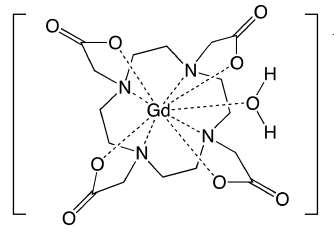

Gd-DOTA ( [Gd(DOTA)( $\left.\left.\mathrm{H}_{2} \mathrm{O}\right)\right]^{-}$)<smiles>CC1(C)CC(=O)CC(C)(C)N1O</smiles><smiles>CC1CC(O)CC(C)(C)N1C</smiles>

TEMPONE

TEMPOL TEMPONE, and TEMPOL 
cal Industry Co., Ltd. (Tokyo, Japan), TEMPOL from SigmaAldrich Chemical Co. (St. Louis, MO, U.S.A.), and gadolinium chloride hexahydrate from Wako Pure Chemical Industries, Ltd. (Osaka, Japan). Paramagnetic lithium phthalocyanine (LiPc) was synthesized electrochemically in our laboratory following the method reported in the literature. ${ }^{10)}$ TEMPONE and TEMPOL were dissolved in pure water at a concentration of $1.0 \times 10^{-2} \mathrm{~mol} / \mathrm{L}$ and stored at $-20^{\circ} \mathrm{C}$. Gadolinium chloride was dissolved in $2.5 \times 10^{-4} \mathrm{~mol} / \mathrm{L} \mathrm{HCl}$ at a concentration of $4 \mathrm{~mol} / \mathrm{L}$. Meglumine gadopentetate (Gd-DTPA), gadodiamide hydrate (Gd-DTPA-BMA), and meglumine gadoterate (GdDOTA) were purchased from Bayer HealthCare (Osaka, Japan), Daiichi Sankyo Co., Ltd. (Tokyo, Japan), and TERUMO Co. (Tokyo, Japan), respectively, as injection solutions of MRI contrast agent. All other reagents were of the highest purity commercially available. Pure water was freshly prepared with a Millipore Simplicity UV (Billerica, MA, U.S.A.).

Rat Plasma SPF rat (Wister, male) was supplied by Kyudo Co., Ltd. (Tosu, Japan), and were fed a commercial diet (CE-2, CLEA Japan, Inc., Tokyo, Japan) and water ad libitum in a temperature- and humidity-controlled room until experiment. The rat $(450 \mathrm{~g})$ was anesthetized with pentobarbital sodium salt $(70 \mathrm{mg} / \mathrm{kg}$-body weight (b.w.), intraperitoneally (i.p.)), and blood was collected from the heart into heparinized syringe. Plasma was separated from blood cells by centrifugation $\left(400 \times \boldsymbol{g}, 15 \mathrm{~min}, 4^{\circ} \mathrm{C}\right)$. Rat was treated and handled according to recommendations for the handling of laboratory animals for biomedical research, which complied with the Committee on the Safety and Handling Regulations for Laboratory Animal Experiments in our institute.

ESR Experiment TEMPONE, TEMPOL, gadolinium chloride, and MRI contrast agents were diluted with $20 \mathrm{mmol} / \mathrm{L} \quad N$-(2-hydroxyethyl)piperazine- $N$ '-2-ethanesulfonic acid (HEPES) buffer, pH 7.4, to appropriate concentrations. Fifty microliters of a diluted solution of TEMPONE or TEMPOL was mixed with $50 \mu \mathrm{L}$ of a diluted solution of MRI contrast agent or gadolinium chloride. In some experiments, a solution of MRI contrast agent $(10 \mu \mathrm{L})$ and a solution of TEMPONE $(10 \mu \mathrm{L})$ were mixed with $80 \mu \mathrm{L}$ of rat plasma. Fourteen microliters of the mixture was taken into a $100 \mu \mathrm{L}$ disposable micropipette (Drummond Scientific Co., Broomall, PA, U.S.A.), placed in an ESR sample tube, and set in an ESR cavity. X-Band ESR spectra were recorded at $100 \mathrm{kHz}$ field modulation with a JEOL JES-FR80 spectrometer (JEOL Ltd., Tokyo, Japan). Modulation amplitudes were $0.01 \mathrm{mT}$ for TEMPONE and $0.63 \mathrm{mT}$ for TEMPOL. The microwave power was $4.0 \mathrm{~mW} . \mathrm{Mn}^{2+}$ was used as an internal standard of the ESR cavity. For the ESR measurement under $\mathrm{N}_{2}$ atmosphere, $14 \mu \mathrm{L}$ of the mixture was taken in a gas-permeable polytetrafluoroethylene (PTFE) tube (1.524 mm i.d., $1.626 \mathrm{~mm} \mathrm{o.d.),} \mathrm{inserted}$ into a quartz tube, and set in an ESR cavity. ESR spectra were recorded under a stream of $\mathrm{N}_{2}$ gas $(99.999 \%)$. ESR spectra were analyzed with a JEOL ESR-IPRITS data system (ver. 6.4.0). Low field signal of nitroxyl radical was used to obtain peak-to-peak line width and signal height. Signal height was corrected with height of $\mathrm{Mn}^{2+}$ signal.

Confirmation of Removal of Dissolved Oxygen Removal of oxygen was confirmed using ESR oximetry with LiPc. ${ }^{10,11)}$ LiPc ( $2 \mathrm{mg}$ ) was suspended in $1 \mathrm{~mL}$ of HEPES buffer. The suspension $(14 \mu \mathrm{L})$ was taken in the PTFE tube, inserted into a quartz tube, and set in an ESR cavity. ESR spectra were recorded repeatedly at $0.5 \mathrm{~min}$ interval under a stream of $\mathrm{N}_{2}$ gas. The removal of dissolved oxygen was evaluated with a decrease of line width of LiPc signal.

Determination of Gadolinium Concentration with ICPAES MRI contrast agents and gadolinium chloride were diluted with $0.1 \mathrm{~mol} / \mathrm{L} \mathrm{HCl}$ to a concentration less than $1 \mathrm{ppm}$, and gadolinium concentration was determined with a Varian 730-ES ICP atomic emission spectrometer (Varian Inc., Palo Alto, CA, U.S.A.). Gadolinium concentrations in this study were based on the ICP-AES analysis.

\section{Results}

Effect of Gd-DTPA on ESR Spectra of Nitroxyl Radicals ESR spectra of aqueous solutions of $50 \mu \mathrm{mol} / \mathrm{L}$ TEMPONE and TEMPOL were recorded at room temperature in the presence of various concentrations of Gd-DTPA. Line widths $\left(\Delta H_{\mathrm{msl}}\right)$ of ESR signals of both nitroxyl radicals were increased with an increasing concentration of Gd-DTPA, in accordance with a decrease of signal height $(h)$ (Figs. 2A, B). The increase in the line width and the decrease in the signal height were more remarkable in TEMPONE than in TEMPOL. The similar responses of TEMPONE signal were observed in the mixture containing $80 \%$ rat plasma, although the signal height was slightly decreased in comparison with the signal height measured in the absence of plasma (Fig. $2 \mathrm{C})$. When the line width was plotted against concentration of Gd-DTPA in the plasma-free mixture, a linear relationship was observed up to approximately $50 \mathrm{mmol} / \mathrm{L}$ Gd-DTPA in the mixture (Fig. 3A). Correlation coefficients $(r)$ were 0.998 for TEMPONE and 0.996 for TEMPOL. The slope of the line for TEMPONE $(4.97 \mu \mathrm{T} \cdot \mathrm{L} / \mathrm{mmol})$ was 1.4 -times greater than that for TEMPOL $(3.51 \mu \mathrm{T} \cdot \mathrm{L} / \mathrm{mmol})$. These observations indicate that TEMPONE is better than TEMPOL as a probe of GdDTPA estimation.

Measurement of line width was difficult at a higher concentration of Gd-DTPA because decreased signal height made the $S / N$ ratio very small, as shown in Fig. 2. Area of integrated signal $(A)$ of ESR (double integrated value of ESR signal) is related to $h$ and $\Delta H_{\mathrm{msl}}$ as follows:

$$
A=c\left(\Delta H_{\mathrm{msl}}\right)^{2} h
$$

where $c$ is a constant depending on line shape (Gaussian and Lorentzian). ${ }^{12)}$ This indicates that line width should be proportional to $h^{-1 / 2}$ unless the line shape changes. Thus, $h^{-1 / 2}$ was plotted against concentration of Gd-DTPA (Fig. 3B). The values of $h^{-1 / 2}$ also showed linear relationships to Gd-DTPA concentration for both nitroxyl radicals.

Effect of Oxygen on the Response of TEMPONE Line Width to Gd-DTPA Concentration Concentration of GdDTPA in the plasma was usually less than $1 \mathrm{mmol} / \mathrm{L}$ when Gd-DTPA was intravenously injected into a man at an approved dose in Japan $(0.2 \mathrm{mmol} / \mathrm{kg}$ body weight; Package Insert of Magnevist ${ }^{\circledR}$ injection, Bayer). Therefore, higher response should be necessary at low concentration of GdDTPA to monitor its concentration in the plasma. A comparison of the slopes of calibration curves of TEMPONE and TEMPOL suggests that a probe with an intrinsic line width that is narrow should give a high response to the concentration of Gd-DTPA. Dissolved oxygen is known to broaden the signal of nitroxyl radicals. ${ }^{13-15)}$ Therefore, the effect of $\mathrm{N}_{2}$ atmosphere on the slope of calibration curve was examined with 


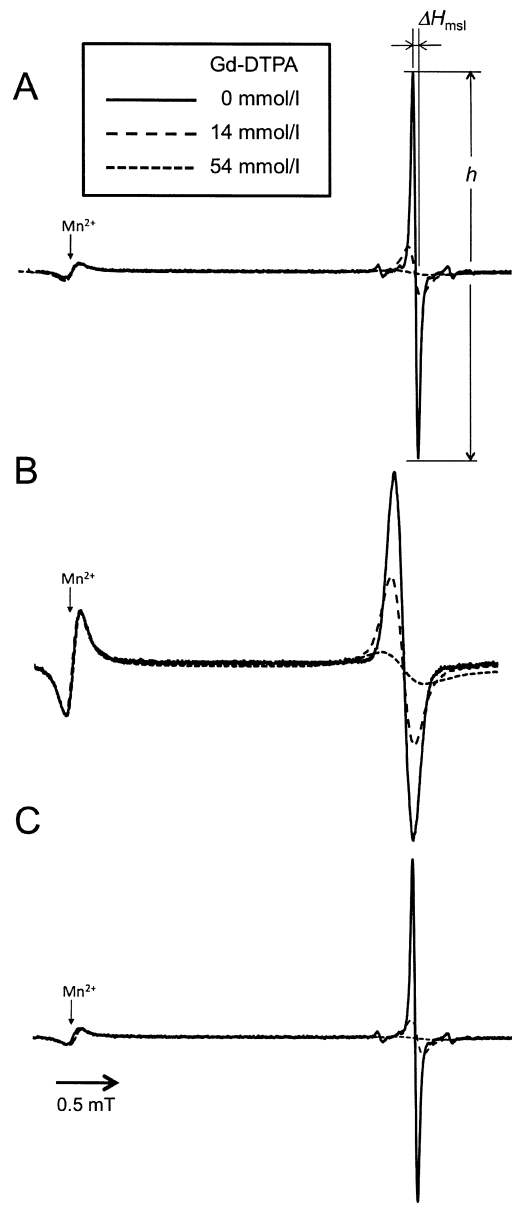

Fig. 2. Effect of Gd-DTPA on ESR Spectra of TEMPONE and TEMPOL

ESR spectra of TEMPONE (A) and TEMPOL (B) in aqueous solutions $(50 \mu \mathrm{mol} / \mathrm{L})$ were recorded in the presence of various concentrations of Gd-DTPA Although each peak of triplet signal of the nitroxyl radical was broadened and shortened equally in the presence of Gd-DTPA, low field peak of the nitroxyl triplet signal was recorded with $\mathrm{Mn}^{2+}$ signal at full sweep width to clarify the alteration of line width of the signal. ESR spectra of $50 \mu \mathrm{mol} / \mathrm{L}$ TEMPONE in $80 \%$ rat plasma was also recorded in the presence of various concentration of Gd-DTPA (C). The concentration of Gd-DTPA in the mixture is indicated in the figure. Spectrometer settings were time constant, $0.03-3.0 \mathrm{~s}$; and sweep time, $2-30 \mathrm{~min}$.

TEMPONE. It was confirmed that dissolved oxygen in the sample solution was replaced with $\mathrm{N}_{2}$ within 10 min under our conditions (Fig. 4, inset). As shown in Fig. 4, the slope obtained under $\mathrm{N}_{2}$ atmosphere was the same as that obtained under air saturation, although $\mathrm{N}_{2}$ atmosphere decreased the line width of TEMPONE from $39.1 \mu \mathrm{T}$ to $31.8 \mu \mathrm{T}$. The results suggest that decrease in the line width by $\mathrm{N}_{2}$ is insufficient to increase the slope of calibration curve obtained with TEMPONE.

The $S / N$ Ratio of ESR Spectrum and the Precision of ESR Parameters Decreased $S / N$ ratio at higher concentration of Gd-DTPA made measurement of line width difficult, as mentioned above. To increase the $S / N$ ratio, increase of TEMPONE concentration is necessary, but excessive increase of TEMPONE concentration causes line-broadening by spinspin interaction of TEMPONE itself, ${ }^{15)}$ which may blunt the response to Gd-DTPA concentration. Therefore, the effects of increased concentration of TEMPONE on the $S / N$ ratio and line width of ESR signal were examined. The increase of concentration of TEMPONE to $200 \mu \mathrm{mol} / \mathrm{L}$ remarkably increased the $\mathrm{S} / \mathrm{N}$ ratio of TEMPONE signal measured in the presence
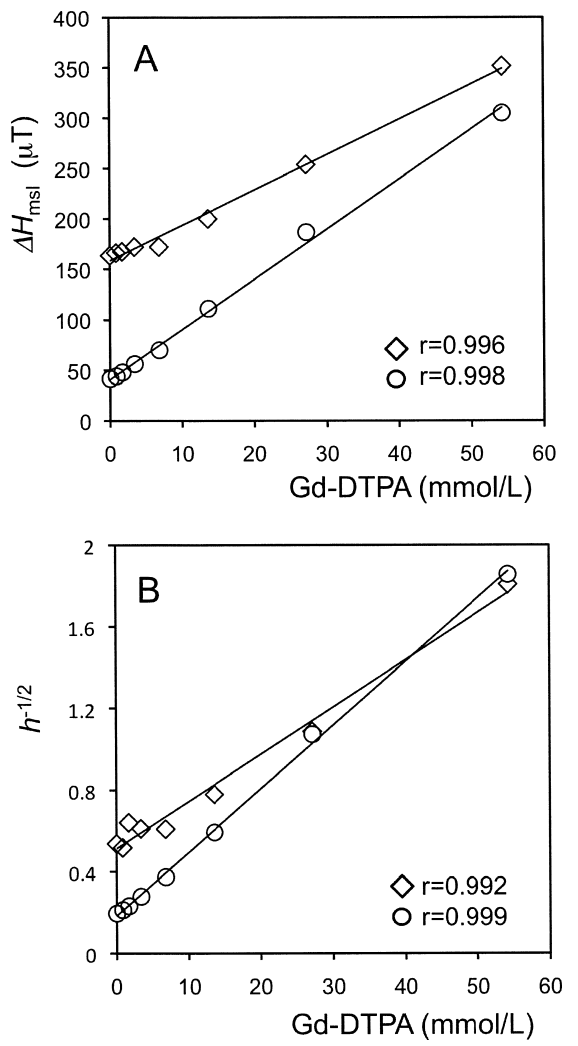

Fig. 3. Relationships of ESR Parameters of Nitroxyl Radicals to GdDTPA Concentration

(A) ESR spectra of TEMPONE (circle) and TEMPOL (diamond) were recorded in the presence of various concentrations of Gd-DTPA, as shown in Fig. 1. The peak-to-peak line width $\left(\Delta H_{\mathrm{msl}}\right)$ of low field signal was plotted against concentration of Gd-DTPA in the mixture. The slopes were $4.97 \mu \mathrm{T} \cdot \mathrm{L} / \mathrm{mmol}$ for TEMPONE and $3.51 \mu \mathrm{T} \cdot \mathrm{L} / \mathrm{mmol}$ for TEMPOL. (B) The reciprocal square root of height $\left(h^{-1 / 2}\right)$ of low field signal was plotted against concentration of Gd-DTPA in the mixture. Similar results were obtained from at least three independent experiments.

of $54 \mathrm{mmol} / \mathrm{L}$ Gd-DTPA (Table 1). On the other hand, the intrinsic line width was increased slightly, but the increase did not affect the response to the concentration of Gd-DTPA. These results indicate that estimation of Gd-DTPA could be carried out at a higher $S / N$ ratio in $200 \mu \mathrm{mol} / \mathrm{L}$ TEMPONE solution.

To compare the magnitude of experimental error of the line width with that of the signal height, $14 \mu \mathrm{L}$ of $200 \mu \mathrm{mol} / \mathrm{L}$ TEMPONE solution was repeatedly taken, set at the same position in the ESR cavity, and measured with ESR. Relative standard deviation (RSD) of the signal height was $8.7 \%$ $(n=15)$, whereas RSD of the line width was $1.7 \%(n=15)$. However, RSD of the signal height $(3.6 \%, n=15)$ was smaller than that of the line width $(5.7 \%, n=15)$ in the presence of $54 \mathrm{mmol} / \mathrm{L}$ Gd-DTPA. This observation indicates that the plot of $h^{-1 / 2}$ against concentration of Gd contrast agents should be useful at relatively high concentration of Gd contrast agents if careful experiment is carried out.

Calibration Curve Obtained with Various Gadolinium Compounds To examine whether or not the ESR method is applicable to other Gd contrast agents, gadolinium complexes with various ligands were used. Gd-DOTA itself gave a very broad ESR signal over a wide range of magnetic fields (Fig. 5A), whereas Gd-DTPA-BMA and gadolinium chloride gave no ESR signal as Gd-DTPA. Measurement of the line width of 


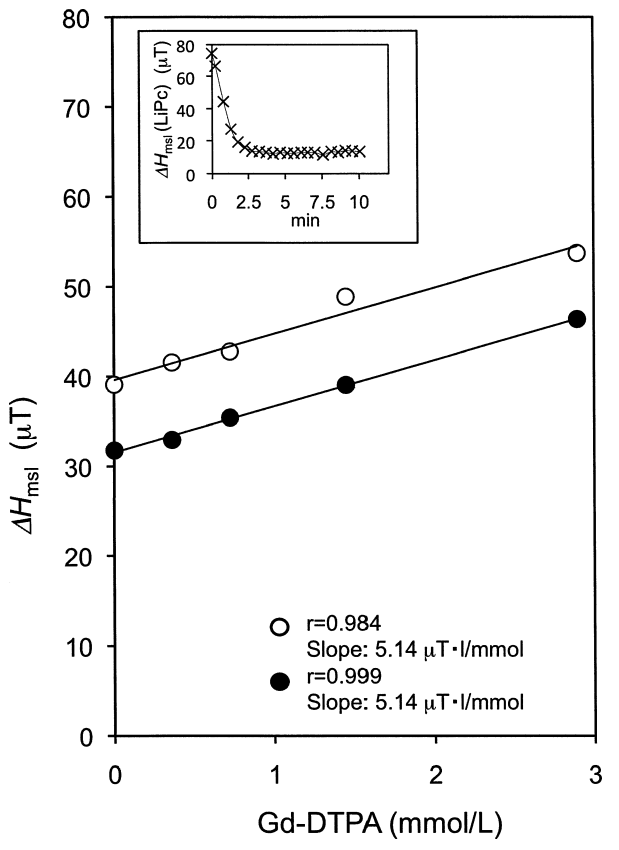

Fig. 4. Effect of Removal of Dissolved Oxygen on the Response of ESR Line Width of TEMPONE to the Concentration of Gd-DTPA

Inset indicates replacement of dissolved oxygen with $\mathrm{N}_{2}$ as confirmed with narrowing of ESR signal of LiPc (oximetry probe). ESR spectra of $50 \mu \mathrm{mol} / \mathrm{L}$ TEM PONE were recorded in the presence of various concentrations of Gd-DTPA in air (open circle) or $\mathrm{N}_{2}$ (filled circle) atmosphere. The spectra under $\mathrm{N}_{2}$ atmosphere were recorded $10 \mathrm{~min}$ after the start of $\mathrm{N}_{2}$ gas flow. The peak-to-peak line width $\left(\Delta H_{\mathrm{msl}}\right)$ of low field peak of ESR spectrum was plotted against the concentration of Gd-DTPA in the mixture.

TEMPONE was possible up to at least $40 \mathrm{mmol} / \mathrm{L}$ Gd-DOTA, although the broad signal tilted the baseline of TEMPONE signal (Fig. 5B).

As shown in Fig. 6, the gadolinium compounds gave a good linear relationship as obtained with Gd-DTPA. Measurement of line width lacked accuracy a little at higher concentration of Gd-DOTA owing to baseline distortion caused by the signal of Gd-DOTA itself. The slopes of the lines were slightly different. The slopes for Gd-DTPA-BMA $(6.22 \mu \mathrm{T} \cdot \mathrm{L} / \mathrm{mmol})$ and Gd-DOTA $(6.62 \mu \mathrm{T} \cdot \mathrm{L} / \mathrm{mmol})$ were steeper than the slope for Gd-DTPA $(5.45 \mu \mathrm{T} \cdot \mathrm{L} / \mathrm{mmol})$, whereas the slope for gadolinium chloride $(4.94 \mu \mathrm{T} \cdot \mathrm{L} / \mathrm{mmol})$ was less steep than that for $\mathrm{Gd}$ DTPA. This indicates that calibration should be carried out with each standard compound. A high correlation coefficient was also obtained in the plot of $h^{-1 / 2}$ against concentrations of Gd-DTPA $(r=0.995)$, Gd-DTPA-BMA $(r=0.999)$, Gd-DOTA $(r=0.997)$, and gadolinium chloride $(r=0.999)$.
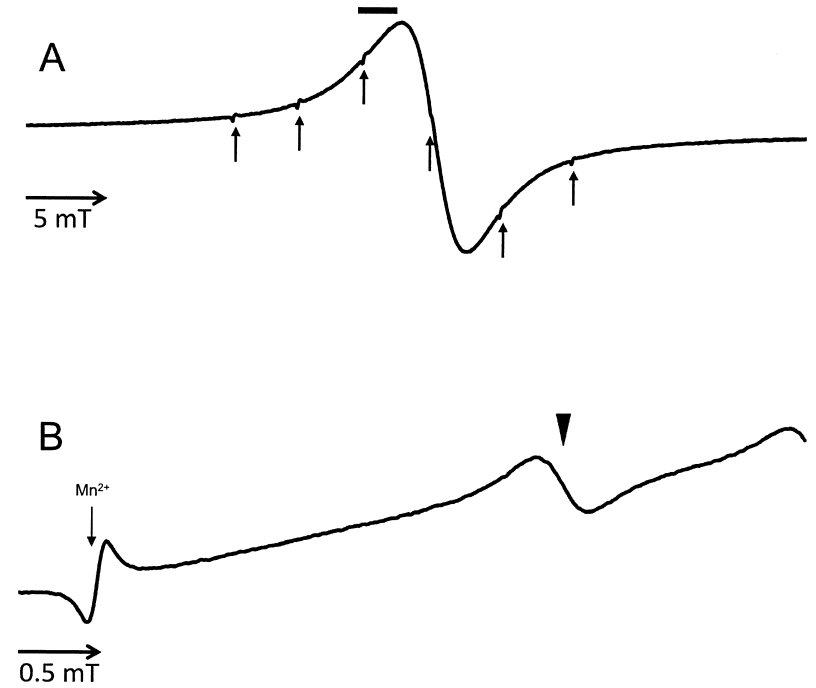

Fig. 5. ESR Signal of Gd-DOTA and Its Effect on ESR Signal of TEMPONE

(A) ESR spectrum of an aqueous solution of $40 \mathrm{mmol} / \mathrm{L}$ Gd-DOTA was recorded. Spectrometer settings were amplitude of field modulation, $0.01 \mathrm{mT}$; magnetic field, $335.6 \pm 50.0 \mathrm{mT}$; time constant, $1.0 \mathrm{~s}$; and sweep time, $15 \mathrm{~min}$. Sextet signal of $\mathrm{Mn}^{2+}$ is indicated with arrows. A thick bar above the spectrum indicates sweep field of spectrum B. (B) ESR spectrum of $200 \mu \mathrm{mol} / \mathrm{L}$ TEMPONE was recorded in the presence of $40 \mathrm{mmol} / \mathrm{L}$ Gd-DOTA. Spectrometer settings were amplitude of field modulation, $0.01 \mathrm{mT}$; and magnetic field, $333.2 \pm 2.5 \mathrm{mT}$. Arrowhead indicates low field signal of TEMPONE.

\section{Discussion}

In the present study, the effects of gadolinium compounds on the ESR line width of nitroxyl radicals, TEMPONE and TEMPOL, were examined to develop a new method for estimation of Gd contrast agents. In both nitroxyl radicals, linear relationships were obtained at high correlation coefficient. The slope of the line for TEMPONE was 1.4-times greater than that of the line for TEMPOL, indicating that TEMPONE is better than TEMPOL as a probe. The slope varied slightly among Gd contrast agents. Therefore, this method should be available if a calibration curve is produced with the same $\mathrm{Gd}$ contrast agent as the object to be measured. The sample volume was $50 \mu \mathrm{L}$ in this study, but the volume could be reduced because only $14 \mu \mathrm{L}$ was used after mixing of the sample solution with a solution of TEMPONE. The method is simple to apply. Thus, it will potentially become a new method for the estimation of Gd contrast agents after some improvements.

It is hard to obtain the line width accurately without a data analyzing system. In this study, a linear relationship was also obtained in a plot of $h^{-1 / 2}$ against concentration of Gd-DTPA. Although the measurement of signal height is easier than the measurement of line width, signal height tends to include experimental error. Signal height should depend on not only

Table 1. Effects of TEMPONE Concentration on $S / N$ Ratio of ESR Signal and ESR Response to Gd-DTPA Concentration

\begin{tabular}{cccc}
\hline \hline TEMPONE conc. ${ }^{a}(\mu \mathrm{mol} / \mathrm{L})$ & Intrinsic line $\operatorname{width}^{b}(\mu \mathrm{T})$ & $S / N$ ratio of signal ${ }^{c)}$ & $\begin{array}{c}\text { Relative response to Gd-DTPA } \\
\text { concentration }^{d}\end{array}$ \\
\hline 50 & 39.1 & 7.9 & 1.00 \\
100 & 42.7 & 15.1 & 0.99 \\
200 & 42.8 & 24.6 & 1.03 \\
\hline
\end{tabular}

a) Concentration for ESR measurement. b) Peak-to-peak line width of low field signal in the absence of Gd-DTPA. c) $S / N$ ratio of TEMPONE ESR spectrum recorded at $3.0 \mathrm{~s}$ time constant in the presence of $54 \mathrm{mmol} / \mathrm{L}$ Gd-DTPA. $d$ ) Relative slope in a plot of $\Delta H_{\mathrm{msl}}$ of TEMPONE signal against concentration of Gd-DTPA. The data were expressed as the ratio of each slope to the slope obtained at $50 \mu \mathrm{mol} / \mathrm{L}$ TEMPONE. 

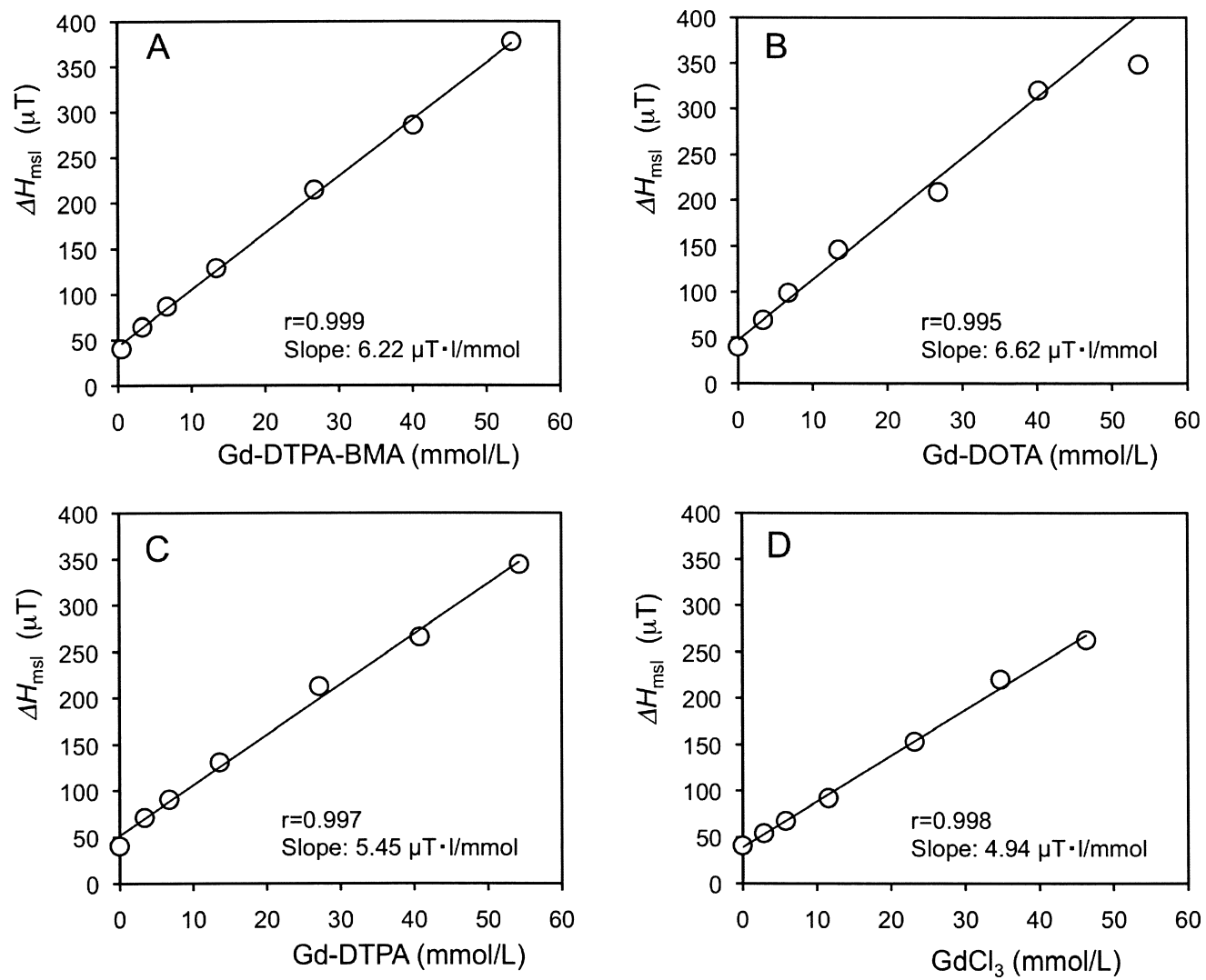

Fig. 6. Effect of Ligand of Gd Complexes on the Response of $\Delta H_{\mathrm{msl}}$ of TEMPONE to the Gd Concentration

ESR spectra of $200 \mu \mathrm{mol} / \mathrm{L}$ TEMPONE solution were recorded in the presence of various concentrations of Gd-DTPA-BMA (A), Gd-DOTA (B), Gd-DTPA (C), and gadolinium chloride (D). The line width of TEMPONE was plotted against Gd concentrations in the mixtures.

concentration of radicals but also volume, dielectric constant, and shape of sample, as well as position of sample in the ESR cavity, among others. Therefore, in the present study, the sample was diluted with the same solvent (a HEPES-buffered solution), $14 \mu \mathrm{L}$ of a sample solution was taken as accurately as possible, and the position of the sample was carefully adjusted at the same position in the ESR cavity every time. The magnitude of RSD of the signal height remarkably smaller than that of the line width in the presence of $54 \mathrm{mmol} / \mathrm{L} \mathrm{Gd}$ DTPA, although RSD of the signal height was larger than that of the line width in the absence of the Gd contrast agents. In our laboratory, a high correlation coefficient was obtained in the plot of $h^{-1 / 2}$ against concentrations of Gd-DTPA $(r=0.995)$, Gd-DTPA-BMA $(r=0.999)$, Gd-DOTA $(r=0.997)$, and gadolinium chloride $(r=0.999)$. Thus, the plot of $h^{-1 / 2}$ against concentrations of Gd compounds could be useful if careful experiments were carried out taking constant volume of sample solution and setting the sample at the same position in the cavity at every measurement.

Gd-DOTA itself gave a broad ESR signal. This suggests that Gd-DOTA could be estimated using its ESR signal. However, it took $15 \mathrm{~min}$ to record the ESR signal of $40 \mathrm{mmol} / \mathrm{L}$ of this agent (Fig. 5) because of insufficient $S / N$ ratio of the signal. Therefore, although estimation of Gd-DOTA concentration may also be possible on the basis of the height of the Gd-DOTA signal, it would be limited in the range of very high concentration.

Some difficulties would be expected when this method is applied to the measurement of concentration of Gd contrast agents in the plasma. The plasma contains reducing agents such as ascorbic acid. The concentration of ascorbic acid is reportedly $10-30 \mu \mathrm{mol} / \mathrm{L}$ in human serum. ${ }^{16,17)}$ Many reports including ours have demonstrated that nitroxyl group of TEMPONE and TEMPOL was easily reduced by ascorbic acid. ${ }^{18-20)}$ Indeed, the signal of TEMPONE decreased in the presence of rat plasma (Fig. 2C). Decline of ESR signal of TEMPONE would interfere with the measurement of line width of TEMPONE signal. Addition of ascorbate oxidase should resolve this problem. Separately, it has been reported that a pyrrolidine nitroxyl, such as 3-carbamoyl-PROXYL, was more resistant to ascorbic acid than a piperidine nitroxyl, such as TEMPONE, ${ }^{18-20)}$ although the intrinsic line width of pyrrolidine nitroxyl was larger than that of TEMPONE. Use of pyrrolidine nitroxyl instead of TEMPONE may be another solution to prevent decline of probe signal. The blood may also contain paramagnetic metal, such as Fe(III) complexes, but the concentration of such compounds should be very low. A standard addition method may avoid the problem, if some interactions take place.

To apply this method to monitor levels of $\mathrm{Gd}$ contrast agents in the plasma, the main problem may be insufficient response to the concentration of the agents. The concentration of Gd contrast agents should be at most $1 \mathrm{mmol} / \mathrm{L}$ in the plasma in clinical use, as described in Results. The $1 \mathrm{mmol} / \mathrm{L}$ increment in Gd-DTPA concentration caused an increment of about $5.0-5.5 \mu \mathrm{T}$ in the line width, as calculated from the slope of calibration curves. This increase was only $12-13 \%$ of the intrinsic line width of TEMPONE. Removal of $\mathrm{O}_{2}$ by $\mathrm{N}_{2}$ gas 
was insufficient to increase the response. Therefore, the application of this method should be, at present, limited to the relatively rough estimation of Gd contrast agents, for example, estimation of the amount of synthesized agents.

Acknowledgments This study was supported by a Grantin-Aid for Scientific Research (No. 22590052) from the Japan Society for the Promotion of Science. We express our deep thanks to Professor Masahiro Sakata, Institute for Environmental Sciences, University of Shizuoka, for measurement of Gd concentration with ICP-AES.

\section{References}

1) Laniado M., Weinmann H. J., Schörner W., Felix R., Speck U., Physiol. Chem. Phys. Med. NMR, 16, 157-165 (1984).

2) Runge V. M., Carollo B. R., Wolf C. R., Nelson K. L., Gelblum D. Y., Radiographics, 9, 929-958 (1989).

3) Greco A., McNamara M. T., Lanthiez P., Quay S. C., Michelozzi G., Radiology, 176, 451-456 (1990).

4) Bousquet J. C., Saini S., Stark D. D., Hahn P. F., Nigam M., Wittenberg J., Ferrucci J. T. Jr., Radiology, 166, 693-698 (1988).

5) Gibby W. A., Gibby K. A., Gibby W. A., Invest. Radiol., 39, 138142 (2004).

6) Frame E. M. S., Uzgiris E. E., Analyst, 123, 675-679 (1998).

7) Lai C. S., Froncisz W., Hopwood L. E., Biophys. J., 52, 625-628 (1987).
8) Takeshita K., Hamada A., Utsumi H., Free Radic. Biol. Med., 26, 951-960 (1999).

9) Magin R. L., Morse P. D. 2nd, Biochim. Biophys. Acta, 760, 357362 (1983).

10) Afeworki M., Miller N. R., Devasahayam N., Cook J., Mitchell J. B., Subramanian S., Krishna M. C., Free Radic. Biol. Med., 25, 72-78 (1998).

11) Takeshita K., Kawaguchi K., Fujii-Aikawa K., Ueno M., Okazaki S., Ono M., Krishna M. C., Kuppusamy P., Ozawa T., Ikota N., Cancer Res., 70, 4133-4140 (2010).

12) Chesnut D. B., J. Magn. Reson., 25, 373-374 (1977).

13) Backer J. M., Budker V. G., Eremenko S. I., Molin Y. N., Biochim. Biophys. Acta, 460, 152-156 (1977).

14) Glockner J. F., Swartz H. M., Pals M. A., J. Cell. Physiol., 140, 505-511 (1989).

15) Jost P., Grifith O. H., "Methods in Pharmacology," Vol. 2, Chap.7, ed. by Chignell C. F., Appleton-Century-Crofts, New York, 1972, pp. $223-276$.

16) Rivers J. M., Huang E. D., Dodds M. L., J. Nutr., 81, 163-168 (1963).

17) Pearson W. N., Am. J. Clin. Nutr., 20, 514-527 (1967).

18) Couet W. R., Brasch R. C., Sosnovsky G., Lukazo J., Prakash I., Gnewuch C. T., Tozer T. N., Tetrahedron, 41, 1165-1172 (1985).

19) Morris S., Sosnovsky G., Hui B., Huber C. O., Rao N. U. M., Swartz H. M., J. Pharm. Sci., 80, 149-152 (1991).

20) Okazaki S., Mannan M. A., Sawai K., Masumizu T., Miura Y., Takeshita K., Free Radic. Res., 41, 1069-1077 (2007). 